\title{
Educação e a
}

Apropriação e

Reconstrução do

\section{Conhecimento}

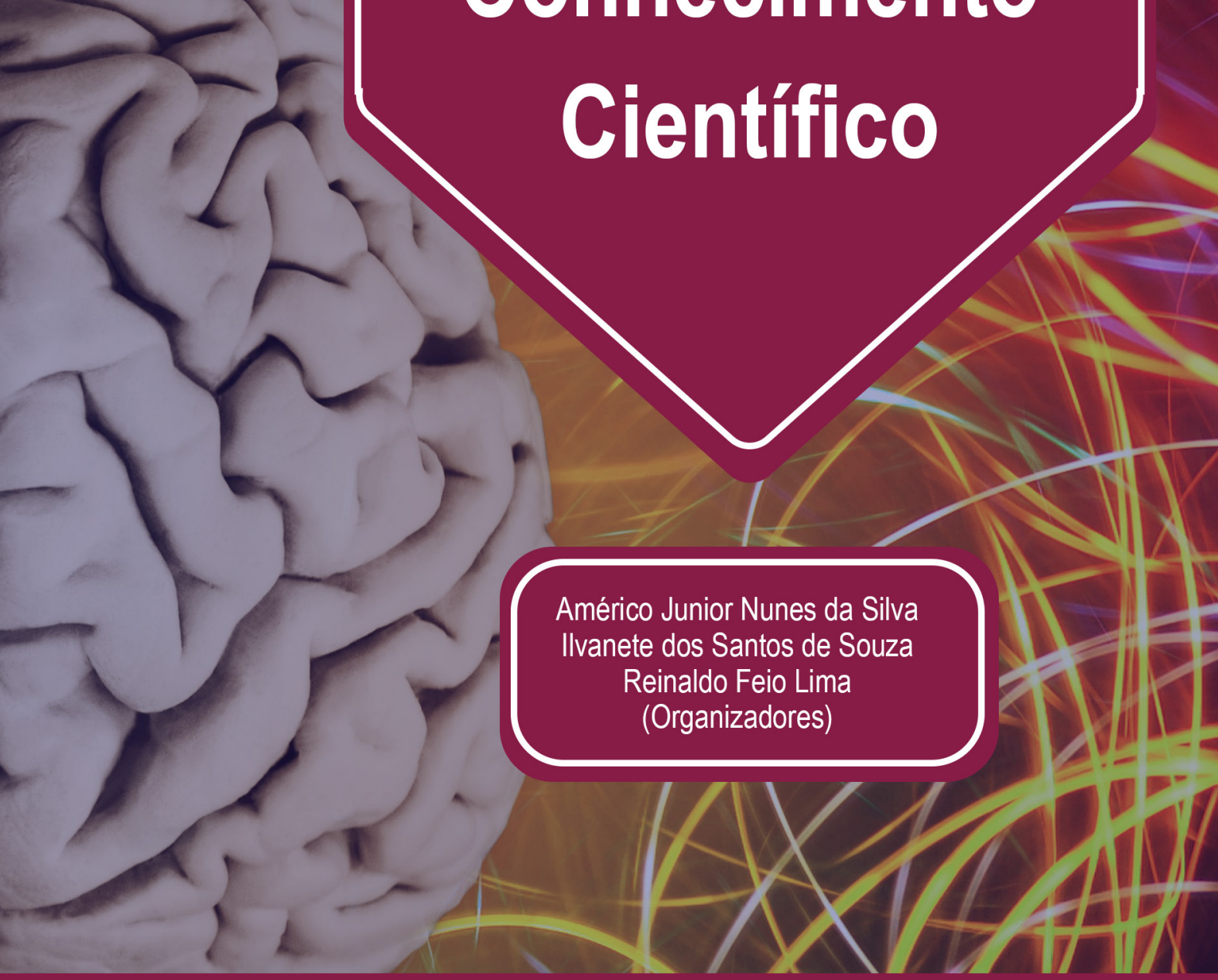

Qגitena

Ano 2020 


\section{Educação e a}

Apropriação e

Reconstrução do

\section{Conhecimento}

\section{Científico}


Editora Chefe

Prof $^{a}$ Dr $^{\mathrm{a}}$ Antonella Carvalho de Oliveira

Assistentes Editoriais

Natalia Oliveira

Bruno Oliveira

Flávia Roberta Barão

Bibliotecária

Janaina Ramos

Projeto Gráfico e Diagramação

Natália Sandrini de Azevedo

Camila Alves de Cremo

Luiza Alves Batista

Maria Alice Pinheiro

Imagens da Capa

Shutterstock

Edição de Arte

Luiza Alves Batista

Revisão

Os Autores
2020 by Atena Editora

Copyright (c) Atena Editora

Copyright do Texto (c) 2020 Os autores

Copyright da Edição (c) 2020 Atena Editora

Direitos para esta edição cedidos à Atena

Editora pelos autores.

Todo o conteúdo deste livro está licenciado sob uma Licença de Atribuição Creative Commons. Atribuição-Não-ComercialNãoDerivativos 4.0 Internacional (CC BY-NC-ND 4.0).

O conteúdo dos artigos e seus dados em sua forma, correção e confiabilidade são de responsabilidade exclusiva dos autores, inclusive não representam necessariamente a posição oficial da Atena Editora. Permitido o download da obra e o compartilhamento desde que sejam atribuídos créditos aos autores, mas sem a possibilidade de alterá-la de nenhuma forma ou utilizá-la para fins comerciais.

Todos os manuscritos foram previamente submetidos à avaliação cega pelos pares, membros do Conselho Editorial desta Editora, tendo sido aprovados para a publicação.

A Atena Editora é comprometida em garantir a integridade editorial em todas as etapas do processo de publicação. Situações suspeitas de má conduta científica serão investigadas sob o mais alto padrão de rigor acadêmico e ético.

\section{Conselho Editorial}

Ciências Humanas e Sociais Aplicadas

Prof. Dr. Alexandre Jose Schumacher - Instituto Federal de Educação, Ciência e Tecnologia do Paraná

Prof. Dr. Américo Junior Nunes da Silva - Universidade do Estado da Bahia

Prof. Dr. Antonio Carlos Frasson - Universidade Tecnológica Federal do Paraná

Prof. Dr. Antonio Gasparetto Júnior - Instituto Federal do Sudeste de Minas Gerais

Prof. Dr. Antonio Isidro-Filho - Universidade de Brasília

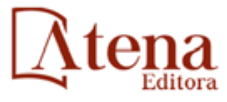


Prof. Dr. Carlos Antonio de Souza Moraes - Universidade Federal Fluminense

Prof $^{a}$ Dr $^{\text {a }}$ Cristina Gaio - Universidade de Lisboa

Prof. Dr. Daniel Richard Sant'Ana - Universidade de Brasília

Prof. Dr. Deyvison de Lima Oliveira - Universidade Federal de Rondônia

Prof $^{\mathrm{a}}$ Dr $^{\mathrm{a}}$ Dilma Antunes Silva - Universidade Federal de São Paulo

Prof. Dr. Edvaldo Antunes de Farias - Universidade Estácio de Sá

Prof. Dr. Elson Ferreira Costa - Universidade do Estado do Pará

Prof. Dr. Eloi Martins Senhora - Universidade Federal de Roraima

Prof. Dr. Gustavo Henrique Cepolini Ferreira - Universidade Estadual de Montes Claros

Prof $^{a} \mathrm{Dr}^{\mathrm{a}}$ Ivone Goulart Lopes - Istituto Internazionele delle Figlie de Maria Ausiliatrice

Prof. Dr. Jadson Correia de Oliveira - Universidade Católica do Salvador

Prof. Dr. Julio Candido de Meirelles Junior - Universidade Federal Fluminense

Prof $^{\mathrm{a}} \mathrm{Dr}^{\mathrm{a}}$ Lina Maria Gonçalves - Universidade Federal do Tocantins

Prof. Dr. Luis Ricardo Fernandes da Costa - Universidade Estadual de Montes Claros

Prof $^{a}$ Dr $^{a}$ Natiéli Piovesan - Instituto Federal do Rio Grande do Norte

Prof. Dr. Marcelo Pereira da Silva - Pontifícia Universidade Católica de Campinas

Prof $^{a}$ Dr $^{a}$ Maria Luzia da Silva Santana - Universidade Federal de Mato Grosso do Sul

Prof ${ }^{a}$ Dr $^{a}$ Paola Andressa Scortegagna - Universidade Estadual de Ponta Grossa

Prof $^{a}$ Dr $^{a}$ Rita de Cássia da Silva Oliveira - Universidade Estadual de Ponta Grossa

Prof. Dr. Rui Maia Diamantino - Universidade Salvador

Prof. Dr. Urandi João Rodrigues Junior - Universidade Federal do Oeste do Pará

Prof $^{a}$ Dr $^{a}$ Vanessa Bordin Viera - Universidade Federal de Campina Grande

Prof. Dr. William Cleber Domingues Silva - Universidade Federal Rural do Rio de Janeiro

Prof. Dr. Willian Douglas Guilherme - Universidade Federal do Tocantins

\section{Ciências Agrárias e Multidisciplinar}

Prof. Dr. Alexandre Igor Azevedo Pereira - Instituto Federal Goiano

Prof $^{a}$ Dr $^{a}$ Carla Cristina Bauermann Brasil - Universidade Federal de Santa Maria

Prof. Dr. Antonio Pasqualetto - Pontifícia Universidade Católica de Goiás

Prof. Dr. Cleberton Correia Santos - Universidade Federal da Grande Dourados

Prof $^{a}$ Dr $^{\mathrm{a}}$ Daiane Garabeli Trojan - Universidade Norte do Paraná

Prof ${ }^{a}$ Dr $^{a}$ Diocléa Almeida Seabra Silva - Universidade Federal Rural da Amazônia

Prof. Dr. Écio Souza Diniz - Universidade Federal de Viçosa

Prof. Dr. Fábio Steiner - Universidade Estadual de Mato Grosso do Sul

Prof. Dr. Fágner Cavalcante Patrocínio dos Santos - Universidade Federal do Ceará

Prof ${ }^{a}$ Dr $^{a}$ Girlene Santos de Souza - Universidade Federal do Recôncavo da Bahia

Prof. Dr. Jael Soares Batista - Universidade Federal Rural do Semi-Árido

Prof. Dr. Júlio César Ribeiro - Universidade Federal Rural do Rio de Janeiro

Prof $^{a}$ Dr $^{a}$ Lina Raquel Santos Araújo - Universidade Estadual do Ceará

Prof. Dr. Pedro Manuel Villa - Universidade Federal de Viçosa

Prof $^{\mathrm{a}}$ Dr $^{\mathrm{a}}$ Raissa Rachel Salustriano da Silva Matos - Universidade Federal do Maranhão

Prof. Dr. Ronilson Freitas de Souza - Universidade do Estado do Pará

Prof $^{a}$ Dr $^{a}$ Talita de Santos Matos - Universidade Federal Rural do Rio de Janeiro

Prof. Dr. Tiago da Silva Teófilo - Universidade Federal Rural do Semi-Árido

Prof. Dr. Valdemar Antonio Paffaro Junior - Universidade Federal de Alfenas

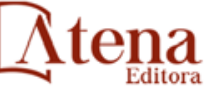

Ano 2020 


\section{Ciências Biológicas e da Saúde}

Prof. Dr. André Ribeiro da Silva - Universidade de Brasília

Prof $^{a}$ Dr $^{a}$ Anelise Levay Murari - Universidade Federal de Pelotas

Prof. Dr. Benedito Rodrigues da Silva Neto - Universidade Federal de Goiás

Prof $^{\mathrm{a}} \mathrm{Dr}^{\mathrm{a}}$ Débora Luana Ribeiro Pessoa - Universidade Federal do Maranhão

Prof. Dr. Douglas Siqueira de Almeida Chaves -Universidade Federal Rural do Rio de Janeiro

Prof. Dr. Edson da Silva - Universidade Federal dos Vales do Jequitinhonha e Mucuri

Prof $^{a}$ Dr $^{a}$ Eleuza Rodrigues Machado - Faculdade Anhanguera de Brasília

Prof $^{a}$ Dr $^{a}$ Elane Schwinden Prudêncio - Universidade Federal de Santa Catarina

Prof $^{\mathrm{a}} \mathrm{Dr}^{\mathrm{a}}$ Eysler Gonçalves Maia Brasil - Universidade da Integração Internacional da Lusofonia Afro-Brasileira

Prof. Dr. Ferlando Lima Santos - Universidade Federal do Recôncavo da Bahia

Prof $^{a}$ Dr $^{a}$ Gabriela Vieira do Amaral - Universidade de Vassouras

Prof. Dr. Gianfábio Pimentel Franco - Universidade Federal de Santa Maria

Prof. Dr. Helio Franklin Rodrigues de Almeida - Universidade Federal de Rondônia

Prof $^{a}$ Dr $^{a}$ lara Lúcia Tescarollo - Universidade São Francisco

Prof. Dr. Igor Luiz Vieira de Lima Santos - Universidade Federal de Campina Grande

Prof. Dr. Jefferson Thiago Souza - Universidade Estadual do Ceará

Prof. Dr. Jesus Rodrigues Lemos - Universidade Federal do Piauí

Prof. Dr. Jônatas de França Barros - Universidade Federal do Rio Grande do Norte

Prof. Dr. José Max Barbosa de Oliveira Junior - Universidade Federal do Oeste do Pará

Prof. Dr. Luís Paulo Souza e Souza - Universidade Federal do Amazonas

Prof $^{a}$ Dr $^{a}$ Magnólia de Araújo Campos - Universidade Federal de Campina Grande

Prof. Dr. Marcus Fernando da Silva Praxedes - Universidade Federal do Recôncavo da Bahia

Prof ${ }^{a} \operatorname{Dr}^{a}$ Maria Tatiane Gonçalves Sá - Universidade do Estado do Pará

Prof $^{a}$ Dr $^{a}$ Mylena Andréa Oliveira Torres - Universidade Ceuma

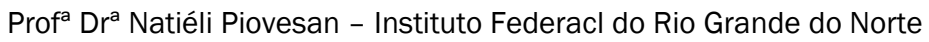

Prof. Dr. Paulo Inada - Universidade Estadual de Maringá

Prof. Dr. Rafael Henrique Silva - Hospital Universitário da Universidade Federal da Grande Dourados

Prof ${ }^{a}$ Dr $^{a}$ Regiane Luz Carvalho - Centro Universitário das Faculdades Associadas de Ensino

Prof $^{a}$ Dr $^{a}$ Renata Mendes de Freitas - Universidade Federal de Juiz de Fora

Prof $^{a}$ Dr $^{\text {a }}$ Vanessa Lima Gonçalves - Universidade Estadual de Ponta Grossa

Prof $^{a}$ Dr $^{\mathrm{a}}$ Vanessa Bordin Viera - Universidade Federal de Campina Grande

\section{Ciências Exatas e da Terra e Engenharias}

Prof. Dr. Adélio Alcino Sampaio Castro Machado - Universidade do Porto

Prof. Dr. Carlos Eduardo Sanches de Andrade - Universidade Federal de Goiás

Prof ${ }^{a}$ Dr $^{a}$ Carmen Lúcia Voigt - Universidade Norte do Paraná

Prof. Dr. Douglas Gonçalves da Silva - Universidade Estadual do Sudoeste da Bahia

Prof. Dr. Eloi Rufato Junior - Universidade Tecnológica Federal do Paraná

Prof $^{a}$ Dr $^{a}$ Érica de Melo Azevedo - Instituto Federal do Rio de Janeiro

Prof. Dr. Fabrício Menezes Ramos - Instituto Federal do Pará

Prof $^{a}$ Dra. Jéssica Verger Nardeli - Universidade Estadual Paulista Júlio de Mesquita Filho

Prof. Dr. Juliano Carlo Rufino de Freitas - Universidade Federal de Campina Grande

Prof $^{\mathrm{a}} \mathrm{Dr}^{\mathrm{a}}$ Luciana do Nascimento Mendes - Instituto Federal de Educação, Ciência e Tecnologia

do Rio Grande do Norte

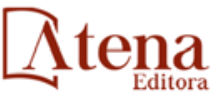

Ano 2020 
Prof. Dr. Marcelo Marques - Universidade Estadual de Maringá

Prof $^{a} D^{a}{ }^{a}$ Neiva Maria de Almeida - Universidade Federal da Paraíba

Prof $^{a}$ Dra $^{a}$ Natiéli Piovesan - Instituto Federal do Rio Grande do Norte

Prof ${ }^{a}$ Dr $^{a}$ Priscila Tessmer Scaglioni - Universidade Federal de Pelotas

Prof. Dr. Takeshy Tachizawa - Faculdade de Campo Limpo Paulista

\section{Linguística, Letras e Artes}

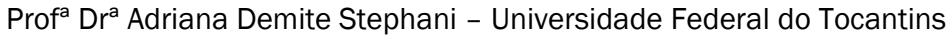

Prof $^{\mathrm{a}} \mathrm{Dr}^{\mathrm{a}}$ Angeli Rose do Nascimento - Universidade Federal do Estado do Rio de Janeiro

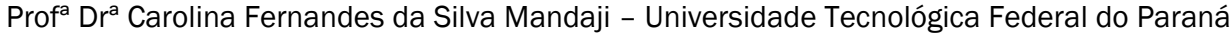

Prof $^{a}$ Dr $^{a}$ Denise Rocha - Universidade Federal do Ceará

Prof. Dr. Fabiano Tadeu Grazioli - Universidade Regional Integrada do Alto Uruguai e das Missões

Prof. Dr. Gilmei Fleck - Universidade Estadual do Oeste do Paraná

Prof $^{a}$ Dr $^{a}$ Keyla Christina Almeida Portela - Instituto Federal de Educação, Ciência e Tecnologia do Paraná

Prof $^{a}$ Dra $^{a}$ Miranilde Oliveira Neves - Instituto de Educação, Ciência e Tecnologia do Pará

Prof $^{a}$ Dr $^{a}$ Sandra Regina Gardacho Pietrobon - Universidade Estadual do Centro-Oeste

Prof $^{a}$ Dr $^{\text {a }}$ Sheila Marta Carregosa Rocha - Universidade do Estado da Bahia

\section{Conselho Técnico Científico}

Prof. Me. Abrãao Carvalho Nogueira - Universidade Federal do Espírito Santo

Prof. Me. Adalberto Zorzo - Centro Estadual de Educação Tecnológica Paula Souza

Prof. Dr. Adaylson Wagner Sousa de Vasconcelos - Ordem dos Advogados do Brasil/Seccional

Paraíba

Prof. Dr. Adilson Tadeu Basquerote Silva - Universidade para o Desenvolvimento do Alto Vale do Itajaí

Prof. Me. Alexsandro Teixeira Ribeiro - Centro Universitário Internacional

Prof. Me. André Flávio Gonçalves Silva - Universidade Federal do Maranhão

Prof $^{\mathrm{a}}$ Ma. Andréa Cristina Marques de Araújo - Universidade Fernando Pessoa

Prof ${ }^{a}$ Dr $^{a}$ Andreza Lopes - Instituto de Pesquisa e Desenvolvimento Acadêmico

Prof ${ }^{a}$ Dr $^{a}$ Andrezza Miguel da Silva - Faculdade da Amazônia

Prof ${ }^{a}$ Ma. Anelisa Mota Gregoleti - Universidade Estadual de Maringá

Prof $^{a}$ Ma. Anne Karynne da Silva Barbosa - Universidade Federal do Maranhão

Prof. Dr. Antonio Hot Pereira de Faria - Polícia Militar de Minas Gerais

Prof. Me. Armando Dias Duarte - Universidade Federal de Pernambuco

Prof ${ }^{a}$ Ma. Bianca Camargo Martins - UniCesumar

Prof $^{a}$ Ma. Carolina Shimomura Nanya - Universidade Federal de São Carlos

Prof. Me. Carlos Antônio dos Santos - Universidade Federal Rural do Rio de Janeiro

Prof. Ma. Cláudia de Araújo Marques - Faculdade de Música do Espírito Santo

Prof ${ }^{a}$ Dra $^{a}$ Cláudia Taís Siqueira Cagliari - Centro Universitário Dinâmica das Cataratas

Prof. Me. Clécio Danilo Dias da Silva - Universidade Federal do Rio Grande do Norte

Prof. Me. Daniel da Silva Miranda - Universidade Federal do Pará

Prof ${ }^{a}$ Ma. Daniela da Silva Rodrigues - Universidade de Brasília

Prof $^{a}$ Ma. Daniela Remião de Macedo - Universidade de Lisboa

Prof ${ }^{a}$ Ma. Dayane de Melo Barros - Universidade Federal de Pernambuco

Prof. Me. Douglas Santos Mezacas - Universidade Estadual de Goiás

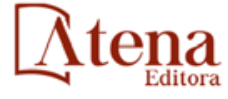


Prof. Me. Edevaldo de Castro Monteiro - Embrapa Agrobiologia

Prof. Me. Eduardo Gomes de Oliveira - Faculdades Unificadas Doctum de Cataguases

Prof. Me. Eduardo Henrique Ferreira - Faculdade Pitágoras de Londrina

Prof. Dr. Edwaldo Costa - Marinha do Brasil

Prof. Me. Eliel Constantino da Silva - Universidade Estadual Paulista Júlio de Mesquita

Prof. Me. Ernane Rosa Martins - Instituto Federal de Educação, Ciência e Tecnologia de Goiás

Prof. Me. Euvaldo de Sousa Costa Junior - Prefeitura Municipal de São João do Piauí

Prof $^{a}$ Ma. Fabiana Coelho Couto Rocha Corrêa - Centro Universitário Estácio Juiz de Fora

Prof. Me. Felipe da Costa Negrão - Universidade Federal do Amazonas

Prof $^{a}$ Dr $^{\text {a }}$ Germana Ponce de Leon Ramírez - Centro Universitário Adventista de São Paulo

Prof. Me. Gevair Campos - Instituto Mineiro de Agropecuária

Prof. Me. Givanildo de Oliveira Santos - Secretaria da Educação de Goiás

Prof. Dr. Guilherme Renato Gomes - Universidade Norte do ParanáProf. Me. Gustavo Krahl Universidade do Oeste de Santa Catarina

Prof. Me. Helton Rangel Coutinho Junior - Tribunal de Justiça do Estado do Rio de Janeiro

Prof $^{a}$ Ma. Isabelle Cerqueira Sousa - Universidade de Fortaleza

Prof $^{a}$ Ma. Jaqueline Oliveira Rezende - Universidade Federal de Uberlândia

Prof. Me. Javier Antonio Albornoz - University of Miami and Miami Dade College

Prof. Me. Jhonatan da Silva Lima - Universidade Federal do Pará

Prof. Dr. José Carlos da Silva Mendes - Instituto de Psicologia Cognitiva, Desenvolvimento Humano e Social

Prof. Me. Jose Elyton Batista dos Santos - Universidade Federal de Sergipe

Prof. Me. José Luiz Leonardo de Araujo Pimenta - Instituto Nacional de Investigación Agropecuaria Uruguay

Prof. Me. José Messias Ribeiro Júnior - Instituto Federal de Educação Tecnológica de Pernambuco

Prof $^{a}$ Dr $^{a}$ Juliana Santana de Curcio - Universidade Federal de Goiás

Prof ${ }^{a}$ Ma. Juliana Thaisa Rodrigues Pacheco - Universidade Estadual de Ponta Grossa

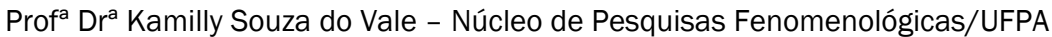

Prof. Dr. Kárpio Márcio de Siqueira - Universidade do Estado da Bahia

Prof $^{\mathrm{a}} \mathrm{Dr}^{\mathrm{a}}$ Karina de Araújo Dias - Prefeitura Municipal de Florianópolis

Prof. Dr. Lázaro Castro Silva Nascimento - Laboratório de Fenomenologia \&

Subjetividade/UFPR

Prof. Me. Leonardo Tullio - Universidade Estadual de Ponta Grossa

Prof ${ }^{a}$ Ma. Lilian Coelho de Freitas - Instituto Federal do Pará

Prof ${ }^{a}$ Ma. Liliani Aparecida Sereno Fontes de Medeiros - Consórcio CEDERJ

Prof $^{a}$ Dr$^{a}$ Lívia do Carmo Silva - Universidade Federal de Goiás

Prof. Dr. Lucio Marques Vieira Souza - Secretaria de Estado da Educação, do Esporte e da Cultura de Sergipe

Prof. Me. Luis Henrique Almeida Castro - Universidade Federal da Grande Dourados

Prof. Dr. Luan Vinicius Bernardelli - Universidade Estadual do Paraná

Prof. Dr. Michel da Costa - Universidade Metropolitana de Santos

Prof. Dr. Marcelo Máximo Purificação - Fundação Integrada Municipal de Ensino Superior

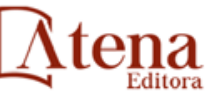

Ano 2020 
Prof. Me. Marcos Aurelio Alves e Silva - Instituto Federal de Educação, Ciência e Tecnologia de São Paulo

Prof ${ }^{a}$ Ma. Maria Elanny Damasceno Silva - Universidade Federal do Ceará

Prof ${ }^{a}$ Ma. Marileila Marques Toledo - Universidade Federal dos Vales do Jequitinhonha e Mucuri

Prof. Me. Ricardo Sérgio da Silva - Universidade Federal de Pernambuco

Prof $^{a}$ Ma. Renata Luciane Polsaque Young Blood - UniSecal

Prof. Me. Robson Lucas Soares da Silva - Universidade Federal da Paraíba

Prof. Me. Sebastião André Barbosa Junior - Universidade Federal Rural de Pernambuco

Prof $^{a}$ Ma. Silene Ribeiro Miranda Barbosa - Consultoria Brasileira de Ensino, Pesquisa e Extensão

Prof $^{a}$ Ma. Solange Aparecida de Souza Monteiro - Instituto Federal de São Paulo

Prof. Me. Tallys Newton Fernandes de Matos - Faculdade Regional Jaguaribana

Prof $^{a}$ Ma. Thatianny Jasmine Castro Martins de Carvalho - Universidade Federal do Piauí

Prof. Me. Tiago Silvio Dedoné - Colégio ECEL Positivo

Prof. Dr. Welleson Feitosa Gazel - Universidade Paulista 


\section{Educação e a apropriação e reconstrução do conhecimento científico}

Editora Chefe: Prof $^{\mathrm{a}} \mathrm{Dr}^{\mathrm{a}}$ Antonella Carvalho de Oliveira

Bibliotecária: Janaina Ramos

Diagramação: Maria Alice Pinheiro

Correção: Mariane Aparecida Freitas

Edição de Arte: Luiza Alves Batista

Revisão: Os Autores

Organizadores: Américo Junior Nunes da Silva Ilvanete dos Santos de Souza

Reinaldo Feio Lima

Dados Internacionais de Catalogação na Publicação (CIP)

E24 Educação e a apropriação e reconstrução do conhecimento científico / Organizadores Américo Junior Nunes da Silva, Ilvanete dos Santos de Souza, Reinaldo Feio Lima. - Ponta Grossa - PR: Atena, 2020.

Formato: PDF

Requisitos de sistema: Adobe Acrobat Reader Modo de acesso: World Wide Web Inclui bibliografia ISBN 978-65-5706-607-2 DOI 10.22533/at.ed.072201512

1. Educação. I. Silva, Américo Junior Nunes da (Organizador). II. Souza, Ilvanete dos Santos de (Organizadora). III. Lima, Reinaldo Feio (Organizador). IV. Título.

CDD 370

Elaborado por Bibliotecária Janaina Ramos - CRB-8/9166

Atena Editora

Ponta Grossa - Paraná - Brasil Telefone: +55 (42) 3323-5493 www.atenaeditora.com.br contato@atenaeditora.com.br 


\section{DECLARAÇÃO DOS AUTORES}

Os autores desta obra: 1. Atestam não possuir qualquer interesse comercial que constitua um conflito de interesses em relação ao artigo científico publicado; 2. Declaram que participaram ativamente da construção dos respectivos manuscritos, preferencialmente na: a) Concepção do estudo, e/ou aquisição de dados, e/ou análise e interpretação de dados; b) Elaboração do artigo ou revisão com vistas a tornar o material intelectualmente relevante; c) Aprovação final do manuscrito para submissão.; 3. Certificam que os artigos científicos publicados estão completamente isentos de dados e/ou resultados fraudulentos. 


\title{
APRESENTAÇÃO
}

Esta obra surge no bojo de uma pandemia: a do novo coronavírus. Contexto marcado pelo distanciamento social e consequentemente a suspensão das atividades presenciais em escolas e universidades. Segundo a Organização das Nações Unidas para a Educação, a Ciência e a Cultura (UNESCO), a pandemia da COVID-19 já impactou os estudos de mais de 1,5 bilhão de estudantes em 188 países. E é nessa conjuntura de um "novo normal" que os autores dessa obra organizam as produções que compõem este volume.

Boaventura de Souza Santos ${ }^{1}$ em sua obra "A cruel pedagogia do vírus" nos apresenta algumas reflexões sobre os desafios desse período emergencial e lança luz sobre as desigualdades sociais evidenciadas por esse panorama. E consequentemente, na Educação, esses aspectos compactuam de algum modo, ao acentuar a exclusão daqueles que não conseguem adequar-se desencadeando impactos no ensino como, por exemplo, acesso a tecnologia, reinvenções metodológicas e a mudança de rotina da sala de aula, dentre outros. O cenário emergencial potencializa os desafios e traz à baila as fragilidades do ensino, ainda em fase de apropriação, pois precisam ser compreendidos, ou seja, as informações carregam intencionalidade.

As discussões realizadas neste volume 1 de "Educação e a Apropriação e Reconstrução do Conhecimento Científico", perpassam pela Educação e seus diferentes contextos e reúnem estudos de autores nacionais e internacionais. Este livro, portanto, reúne trabalhos de pesquisa e experiências em diversos espaços, com o intuito de promover um amplo debate acerca das diversas problemáticas que permeiam o contexto educacional brasileiro. Os capítulos que compõe essa obra abordam, de forma interdisciplinar, a partir da realização de pesquisas, relatos de casos e revisões, problemas e situações comuns do contexto educacional.

Por fim, ao levar em consideração todos os elementos que apresentamos anteriormente, esta obra, a partir das discussões que emergem de suas páginas, constituise enquanto importante leitura para aqueles que fazem Educação no país e que se interessam pelas temáticas aqui discutidas. Nesse sentido, desejamos uma boa leitura a todos e a todas.

\author{
Américo Junior Nunes da Silva \\ Ilvanete dos Santos de Souza \\ Reinaldo Feio Lima
}

1 SANTOS, Boaventura de Sousa. A Cruel Pedagogia do Vírus. Editora Almedina, Portugal. 2020. 


\section{SUMÁRIO}

CAPÍTULO 1

O CARÁTER HUMANITÁRIO PARA A EDUCAÇÃO NA PERSPECTIVA DE NUSSBAUM E DE PAULO FREIRE

Carmem Lucia Albrecht da Silveira

Rosimar Serena Siqueira Esquinsani

DOI 10.22533/at.ed.0722015121

CAPÍTULO 2

PELA DIVERSIDADE NA ESCOLA: PRÁTICAS DE RESISTÊNCIA EM UMA AULA PRETA

Karoline Moreira de Oliveira

Antônio Carlos do Nascimento Osório

DOI 10.22533/at.ed.0722015122

CAPÍTULO 3.

A IMPORTÂNCIA DOS PROJETOS SOCIAIS DESPORTIVOS PARA EVITAR A INSERÇÃO DO ADOLESCENTE NA CRIMINALIDADE

Henrique Freire Simmer

Jose Geraldo Ferreira da Silva

DOI 10.22533/at.ed.0722015123

CAPÍTULO 4

A IMPORTÂNCIA DA INTERVENÇÃO PEDAGÓGICA PARA ALUNOS COM DIFICULDADE DE APRENDIZAGEM NA LEITURA E ESCRITA

Alyne Cristine Domene Martins de Lima

Suzana Sirlene da Silva

Miryan Cristina Buzetti

DOI 10.22533/at.ed.0722015124

CAPÍTULO 5

COMPETÊNCIAS SÓCIOEMOCIONAIS NA BASE NACIONAL COMUM CURRICULAR À LUZ DA PEDAGOGIA HISTÓRICO-CRÍTICA

Edna Mara Corrêa Miranda

Mayrla Pereira Sena Cordeiro

DOI 10.22533/at.ed.0722015125

CAPÍTULO 6. 52

CRIANÇAS REFUGIADAS CONGOLESA NO RIO DE JANEIRO: TRAVESSIAS ATÉ A SALA DE AULA E O AMPARO LEGAL PARA INCLUÍ-LAS

Maicon Salvino Nunes de Almeida

DOI 10.22533/at.ed.0722015126

CAPÍTULO 7

EDUCAÇÃO AMBIENTAL COMO ESTRATÉGIA DE PROMOÇÃO DA SAÚDE MENTAL DA POPULAÇÃO RURAL: BREVES REFLEXÕES SOB A ÓTICA DA PSICOLOGIA AMBIENTAL 
Thiago Almeida Vieira

Iani Dias Lauer-Leite

Maria Mirtes Cortinhas dos Santos

DOI 10.22533/at.ed.0722015127

CAPÍTULO 8

69

FORMAÇÃO CONTINUADA EM LINGUAGEM INFANTIL PARA COORDENADORES PEDAGÓGICOS

Ana Claudia Tenor

DOI 10.22533/at.ed.0722015128

CAPÍTULO 9 76

INTEGRAÇÃO DO ENSINO, PESQUISA E EXTENSÃO PARA O GERENCIAMENTO DE RESÍDUOS SÓLIDOS NA ESCOLA POLITÉCNICA DE PERNANBUCO E SUA VIZINHANÇA

Emilia Rahnemay Kohlman Rabbani

Alyx Diêgo Oliveira Silva

Vitória Fernanda de Paula Lucena

Barbara Virginia Pereira Cavalcanti

Sérgio Peres Ramos da Silva

Maria Conceição da Costa Silva

DOI 10.22533/at.ed.0722015129

CAPÍTULO 10

EXPERIMENTO DIDÁTICO-PEDAGÓGICO: UM CAMINHO PARA A INVESTIGAÇÃO DA GAMIFICAÇÃO NA EDUCAÇÃO

Tiago Bacciotti Moreira

Alvino Moraes de Amorim

Natal dos Santos Soares

DOI 10.22533/at.ed.07220151210

CAPÍTULO 11 106

EDUCAÇÃO E MÍDIAS DIGITAIS: POR UMA METODOLOGIA PARTICIPATIVA, LÚDICA E MULTIMODAL

Fátima Cristina Regis Martins de Oliveira

Mayara Fidalgo Pereira de Barros

Pollyana Rodrigues Pessoa Escalante

DOI 10.22533/at.ed.07220151211

CAPÍTULO 12

DESENVOLVIMENTO COGNITIVO DE CRIANÇAS COM SÍNDROME DE DOWN NA EDUCAÇÃO INFANTIL

Juliana Maria Tozzo

DOI 10.22533/at.ed.07220151212 
INDÍGENAS NOS QUADRINHOS: UM ESTUDO A PARTIR DE AÇÃO DIDÁTICOPEDAGÓGICA

Adriane Pesovento

José Joaci Barboza

DOI 10.22533/at.ed.07220151213

CAPÍTULO 14 138

O USO DE RECURSOS TECNOLÓGICOS NA EDUCAÇÃO ESCOLAR

Cintia Roberta Lara de Andrade

DOI 10.22533/at.ed.07220151214

CAPÍTULO 15 145

INTEGRAÇÃO DAS TIC EM ORGANIZAÇÕES E EMPRESAS EDUCATIVAS: DESENVOLVIMENTO TEÓRICO E DESCRITIVO

José Gómez Galán

DOI 10.22533/at.ed.07220151215

CAPÍTULO 16 156

CARACTERIZACIÓN DE LAS CONCEPCIONES DE LOS DOCENTES UNIVERSITARIOS DE INGENIERÍA SOBRE LA EVALUACIÓN

Fabián Alejandro Buffa

María Basilisa García

Julieta del Hoyo

María Eugenia Victoria Hormaiztegui

Paola Andrea Massa

María Alejandra Fanovich

Lucrecia Ethel Moro

DOI 10.22533/at.ed.07220151216

CAPÍTULO 17 168

MONTESSORI E A NEUROCIÊNCIA: A CONEXÃO NECESSÁRIA NA PRÁTICA DOCENTE Magna Aparecida de Oliveira Pinheiro

Sirlândia Reis de Oliveira Teixeira

Felipa Pacífico Ribeiro de Assis Silveira

DOI 10.22533/at.ed.07220151217

CAPÍTULO 18 180

A TRÍADE DE COMANDOS HÍDRICOS (MÁTER-PÁTER) MAIS IMPORTANTES DO CÉREBRO; FITO,TRI-TALÂMICA,HIPOFISÁRIO

Cícera Paz da Silva

Ítalo Marcos Paz de Andrade

DOI 10.22533/at.ed.07220151218 
CAPÍTULO 19

PRODUÇÃO DO TCC EM UM CURSO DE PEDAGOGIA: EMOÇÕES, SENTIMENTOS E APRENDIZADOS VIVENCIADOS

Selma Barros Daltro de Castro

Luciana Rios da Silva

Rosana Fernandes Falcão

DOI 10.22533/at.ed.07220151219

CAPÍTULO 20 196

TECNOLOGIA NA EDUCAÇÃO

Natal dos Santos Soares

Alvino Moraes de Amorim

Tiago Bacciotti Moreira

DOI 10.22533/at.ed.07220151220

SOBRE OS ORGANIZADORES 215

ÍNDICE REMISSIVO 


\section{CAPÍTULO 16}

\section{CARACTERIZACIÓN DE LAS CONCEPCIONES DE LOS DOCENTES UNIVERSITARIOS DE INGENIERÍA SOBRE LA EVALUACIÓN}

Data de aceite: 01/12/2020

Data de submissão: 03/10/2020

Fabián Alejandro Buffa Grupo de Investigación en la Enseñanza y el Aprendizaje de la Ingeniería (GIEnApl) Universidad Nacional de Mar del Plata Facultad de Ingeniería - Departamento de Ingeniería Química y en Alimentos Mar del Plata - Argentina https://orcid.org/0000-0001-5247-5401

\section{María Basilisa García}

Grupo de Investigación en la Enseñanza y el Aprendizaje de la Ingeniería (GIEnApl) Universidad Nacional de Mar del Plata Facultad de Ingeniería - Departamento de Ingeniería Química y en Alimentos Mar del Plata - Argentina https://orcid.org/0000-0002-4282-6957

\section{Julieta del Hoyo}

Grupo de Investigación en la Enseñanza y el Aprendizaje de la Ingeniería (GIEnApl) Universidad Nacional de Mar del Plata Facultad de Ingeniería - Departamento de Ingeniería Química y en Alimentos Mar del Plata - Argentina https://orcid.org/0000-0003-2578-5126

\section{María Eugenia Victoria Hormaiztegui}

Grupo de Investigación en la Enseñanza y el

Aprendizaje de la Ingeniería (GIEnApl)

Universidad Nacional de Mar del Plata Facultad de Ingeniería - Departamento de Ingeniería Química y en Alimentos Mar del Plata - Argentina Centro de Investigaciones y Desarrollo en
Ciencia y Tecnología de Materiales (CITEMA) Comisión de Investigaciones Científicas de la Provincia de Buenos Aires (CIC) Universidad Tecnológica Nacional - Facultad

Regional La Plata La Plata - Argentina https://orcid.org/0000-0001-9383-3076

Paola Andrea Massa

Grupo de Investigación en la Enseñanza y el Aprendizaje de la Ingeniería (GIEnApl)

Universidad Nacional de Mar del Plata Facultad de Ingeniería - Departamento de Ingeniería Química y en Alimentos Mar del Plata - Argentina https://orcid.org/0000-0001-9794-0645

María Alejandra Fanovich

Grupo de Investigación en la Enseñanza y el Aprendizaje de la Ingeniería (GIEnApl) Universidad Nacional de Mar del Plata Facultad de Ingeniería - Departamento de Ingeniería Química y en Alimentos Mar del Plata - Argentina https://orcid.org/0000-0001-8945-4698

\section{Lucrecia Ethel Moro}

Grupo de Investigación en la Enseñanza y el

Aprendizaje de la Ingeniería (GIEnApl) Universidad Nacional de Mar del Plata Facultad de Ingeniería - Departamento de Ingeniería Química y en Alimentos Mar del Plata - Argentina https://orcid.org/0000-0002-0258-3356

RESUMEN: En el presente trabajo se indagaron las concepciones de los profesores universitarios de ingeniería respecto a la evaluación. Se 
llevó a cabo un estudio descriptivo y con un diseño ex post facto. A partir de un protocolo construido con preguntas indirectas se realizaron entrevistas semi estructuradas a docentes de diferentes asignaturas y de las distintas carreras que ofrece la Facultad. Se extrajeron categorías de respuestas aplicando el método comparativo constante. Se trabajó sobre tres dimensiones: ¿qué se evalúa?, ¿cómo se evalúa? y ¿qué función tiene la evaluación en el aprendizaje? Para cada una de ellas se obtuvieron diferentes tipos de respuesta que muestran concepciones que van desde posturas tradicionales, donde la evaluación no está ligada al aprendizaje, no considera su proceso y solo tiene fines de acreditación; a enfoques más constructivistas que la consideran como reguladora de los aprendizajes y tiene en cuenta todos los aspectos observables de este proceso. Las entrevistas han conseguido proporcionar un espacio en el que los docentes han reflexionado sobre su propia práctica, apareciendo nuevos interrogantes y, en algunos casos, la necesidad de formarse en estos temas. El grupo se propone continuar esta investigación realizando un análisis comparativo entre estas concepciones y otras vinculadas con la enseñanza, recogidas en un trabajo anterior.

PALABRAS CLAVE: concepciones - evaluación - docentes - ingeniería - universidad

\section{CARACTERIZAÇÃO DAS CONCEPÇÕES DOS PROFESSORES UNIVERSITÁRIOS DE ENGENHARIA SOBRE AVALIAÇÃO}

RESUMO: No presente trabalho, foram indagadas as concepções de professores universitários de engenharia sobre avaliação. Foi realizado um estudo descritivo com desenho ex post facto. A partir de um protocolo construído com perguntas indiretas, foram realizadas entrevistas semiestruturadas com professores de diferentes disciplinas e dos diferentes cursos oferecidos pela Faculdade de Engenharia da Universidade Nacional de Mar del Plata. As categorias de resposta foram extraídas aplicando o método comparativo constante. Trabalhou-se em três dimensões: "o que é avaliado?", "como se avalia?" e "que função a avaliação tem na aprendizagem?". Para cada uma delas, foram obtidos diferentes tipos de respostas que mostram concepções que vão desde posturas tradicionais, onde a avaliação não está vinculada à aprendizagem, não considera seu processo e apenas tem fins de acreditação; até abordagens mais construtivistas, que a consideram como reguladora da aprendizagem e leva em consideração todos os aspectos observáveis deste processo. As entrevistas conseguiram proporcionar um espaço no qual os professores refletiram sobre sua própria prática, levantando novas questões e, em alguns casos, a necessidade de capacitação sobre essas questões. O grupo pretende dar continuidade à pesquisa realizando uma análise comparativa entre essas concepções e outras relacionadas ao ensino, coletadas em um trabalho anterior.

PALAVRAS - CHAVE: concepções - avaliação - professores - engenharia - universidade

\section{CHARACTERIZATION OF THE CONCEPTIONS ABOUT EVALUATION OF ENGINEERING UNIVERSITY TEACHERS}

ABSTRACT: In the present work, the conceptions of university engineering professors regarding evaluation were investigated. A descriptive study with an ex post facto design was 
carried out. Based on protocol constructed with indirect questions, semi-structured interviews were carried out with teachers of different subjects and of the different careers offered by the Faculty. Categories of answers were extracted applying the constant comparative method. We worked on three dimensions: "what is evaluated?", "how is it evaluated?" and "what is the role of evaluation in learning?" For each of them, different types of answers were obtained that show conceptions ranging from traditional positions, where evaluation is not linked to learning, does not consider its process and only has accreditation purposes; to more constructivist approaches that consider it as regulatory of learning and takes into account all observable aspects of this process. The interviews have managed to provide a space in which teachers have reflected on their own practice, raising new questions and, in some cases, the need for training on these issues. The group intends to continue this research by making a comparative analysis between these conceptions and others related to teaching, collected in a previous work.

KEYWORDS: conceptions - evaluation - teachers - engineering - university

\section{1। INTRODUCCIÓN}

En el contexto universitario, es bastante frecuente que la evaluación sea considerada un elemento externo a la actividad de aprender, y hasta muchas veces sobredimensionada frente a otros elementos del proceso de enseñanza y de aprendizaje. La evaluación, como reguladora de los aciertos y errores, constituye el motor del proceso educativo y forma una unidad indisoluble junto a la enseñanza y el aprendizaje. (GAIRIN; SANMARTÍ, 1998) No obstante, se observa en muchas aulas que la evaluación condiciona la dinámica de los aprendizajes, a punto tal de ser más relevante la práctica evaluativa que las estrategias de enseñanza, poniéndose en evidencia una tendencia del docente a utilizarla como elemento de control más que como un instrumento que ayude a construir un conocimiento. (THOMAS; BAIN, 1984; SCOULLER, 1999)

Para profundizar sobre esta situación diferentes investigadores indagaron las opiniones que los docentes de distintos niveles educativos tienen sobre la evaluación, de modo de comprender tanto sus concepciones, como así también conocer los recursos y estrategias que utilizan en sus prácticas evaluativas.

Los estudios sobre concepciones realizados desde la psicología cognitiva, asumen la posibilidad de interpretarlas en términos de representaciones y procesos mentales. Estas investigaciones han aportado abundante información que evidencia que el sistema de creencias puesto en práctica a la hora de enseñar y evaluar, es determinante en la configuración del escenario educativo, en la elaboración de propuestas metodológicas y en el uso estrategias evaluativas. (KEMBER, 1997; SAMUELOWICZ; BAIN, 2001, 2002)

Pontes Pedrajas, Poyato López y Oliva Martínez (2016), como resultado de una investigación con docentes en formación, exponen que las concepciones sobre evaluación se pueden relacionar principalmente con dos enfoques educativos antagónicos y otro de carácter intermedio. En un tipo de concepción se encuentran docentes con una visión sobre 
la evaluación de carácter tradicional, que se focaliza en valorar los contenidos adquiridos por los estudiantes, fundamentalmente mediante exámenes escritos; es decir se centra en la función acreditadora de la evaluación. Otro grupo de docentes, mostró una concepción de evaluación con un enfoque que los autores denominaron "innovador", que pone mayor énfasis en el estudiante y en la regulación de sus procesos de aprendizaje. En esta posición, la evaluación se ve como un proceso integral relacionado con la progresión en la construcción de conceptos científicos, el desarrollo de procedimientos y la generación de actitudes favorables hacia el aprendizaje de la ciencia. (KEMBER, 1997; PROSSER et al., 2005) Entre las dos visiones mencionadas (tradicional e innovadora) identificaron un enfoque intermedio, integrado por aquellos docentes en cuyas opiniones aparecen elementos de carácter dual entre estas dos concepciones descriptas. (SAMUELOWICZ; BAIN, 2001) Un estudio realizado por Vilanova, Mateos-Sanz y García (2011) con docentes universitarios encontró también estos tres grandes enfoques, pero mostró que la mayoría de los sujetos entrevistados consideran que al evaluar resulta más importante establecer si el estudiante ha incorporado la información, que saber si ha adquirido las capacidades cognitivas necesarias para relacionar significativamente dicha información. Es decir, se observa claramente la relevancia de la función social de la evaluación como componente normativo (visión conductista, tradicional) por sobre la función pedagógica de la misma (visión constructivista).

Si bien se han realizado diversas investigaciones en torno al tema de evaluación en distintos niveles educativos, es escasa la información recabada en el ámbito de educación superior en carreras de ingeniería. En particular, los protagonistas del proceso de enseñanza y de aprendizaje en ingeniería aún siguen siendo poco estudiados, probablemente, debido a la falta de interacción de este sector educativo con áreas de estudio más pedagógicas. Con el fin de avanzar en esta área vacante, recientemente nuestro grupo ha comenzado a investigar sobre las construcciones personales que tienen los docentes de la Facultad de Ingeniería de la Universidad Nacional de Mar del Plata (UNMDP) acerca de "qué se enseña" y "para qué se enseña". (DEL HOYO et al., 2016). Los resultados de ese estudio permitieron concluir, entre otros conceptos, que los docentes son conscientes de sus limitaciones en el proceso de enseñanza y de aprendizaje. Esto es alentador desde el punto de vista pedagógico dado que permite avanzar en la interacción con el cuerpo docente de modo de poder construir conjuntamente nuevas concepciones sobre el proceso educativo y con ello producir mejoras en la formación de los profesionales que se propone formar.

Específicamente, en el presente trabajo, el enfoque está puesto en caracterizar las concepciones que los docentes de ingeniería tienen sobre la evaluación y compararlas con las obtenidas por los autores mencionados anteriormente. 


\section{I OBJETIVO}

El estudio tiene como objetivo explorar y caracterizar las concepciones sobre evaluación que poseen los docentes de Ingeniería de la UNMDP, vinculadas con su desempeño en la formación de profesionales, particularmente en los aspectos relacionados con "qué se evalúa", "cómo se evalúa" y "qué función tiene la evaluación en el aprendizaje”.

\section{I MÉTODO}

Las actividades realizadas para alcanzar el objetivo planteado se desarrollaron mediante un estudio descriptivo con un diseño ex post facto. La variable en estudio fue la concepción sobre la evaluación de los aprendizajes, analizada en tres dimensiones:

D1: ¿Qué se evalúa?

D2: ¿Cómo se evalúa?

D3: ¿Qué función tiene la evaluación en el aprendizaje?

\subsection{Muestra}

La muestra estuvo compuesta por 17 docentes universitarios voluntarios que se desempeñan en la Facultad de Ingeniería de la UNMDP, con dedicación simple, parcial y exclusiva. Sus disciplinas de formación son: física (1), matemática (2), química (2) e ingeniería (12). La mayoría realiza tareas de investigación en diferentes áreas del conocimiento.

\subsection{Instrumento}

El instrumento para relevar los datos consistió en un cuestionario de preguntas semiestructuradas, con modalidad entrevista. Dado que el objetivo del estudio no fue recoger información explícita, mediada por actitudes proposicionales, sino el de intentar acceder a niveles representacionales de carácter más implícito, se requirió que las concepciones sean indagadas por vías no tan directas. (LIMÓN, 2006; PECHARROMÁN; POZO, 2006) Para ello, se plantearon cuestiones abiertas donde el docente pudiera expresar su opinión. Como resultado de la naturaleza indirecta de las preguntas, la mayoría de las entrevistas no fueron estrictamente limitadas a la enseñanza, sino dirigidas tanto a la enseñanza y el aprendizaje más ampliamente. El protocolo completo (que se reporta en un trabajo anterior, (DEL HOYO et al., 2016) constó de 23 preguntas, que tienen que ver con las características de los docentes de ingeniería y con sus concepciones acerca de la enseñanza. De aquí se trabajó en particular sobre las siguientes tres cuestiones relacionadas con el objetivo de este trabajo:

\section{- ¿Cómo se da cuenta que un alumno aprendió?}

Esta pregunta busca indagar las concepciones de los docentes respecto de qué se evalúa y cómo se evalúa, y por lo tanto, indirectamente, qué significa aprender. Se pueden encontrar opiniones que reflejan una concepción de aprendizaje como un estado, 
evaluando entonces resultados en una sola instancia y con un mismo tipo de instrumento (parcial escrito y presencial, por ejemplo) o si entienden al aprendizaje como un proceso, entonces evalúan en diferentes instancias y con distintos instrumentos (parcial pero también parcialitos, entrevistas, problemas especiales, etc.)

\section{- ¿Qué opina de las evaluaciones a libro abierto? ¿Y de los parciales domi- ciliarios?}

Estas cuestiones apuntan a indagar qué evalúan los docentes y en menor medida, cómo evalúan y qué función tiene la evaluación. Se busca conocer si consideran valiosas las posibilidades de la evaluación a libro abierto y el parcial domiciliario; dado que su objetivo es evaluar si los estudiantes han desarrollado la capacidad de adoptar una actitud proposicional sobre los contenidos trabajados en la asignatura y han adquirido determinadas competencias científicas que le permitan abordar problemas relativamente abiertos. O si por el contrario, les interesa medir el recuerdo de información y/o la habilidad adquirida por parte de los estudiantes para aplicar algoritmos y resolver problemas cerrados, prefiriendo, en este caso, los parciales tradicionales, en formato presencial, con carácter eminentemente individual y a libro cerrado. En cuanto a la función que tiene la evaluación, por medio de estas preguntas se pretende conocer si los docentes interrogados consideran que la evaluación tiene una función exclusivamente acreditadora, que tiene que ver con la certificación del saber, es decir, con el título que otorga la institución educativa; o como una función de control y mejora de los procesos de enseñanza y aprendizaje. En la visión tradicional se da el poder de control a los profesores, en cuanto a la toma de decisiones, la definición de lo que es relevante, bueno o excelente; por otro lado, desde las perspectivas innovadoras se le otorga una función pedagógica en la que la evaluación actúa como reguladora del proceso de enseñanza.

\section{- ¿De qué manera devuelve las evaluaciones?}

Esta pregunta apunta a indagar qué concepción tienen los docentes sobre cómo se evalúa y sobre la función de la evaluación en el aprendizaje. Se busca saber si le dan un lugar relevante como parte fundamental del proceso de aprendizaje y, por lo tanto, la devolución se hace de manera individual de forma tal que se genere un espacio donde el estudiante pueda reflexionar sobre los aprendizajes alcanzados y cuáles son los aspectos que, eventualmente, debería revisar; o, si, por el contrario, se devuelven en forma impersonal, reduciendo a la evaluación a una estrategia para medir resultados de aprendizaje, para calificar y decidir sobre la acreditación de los estudiantes. Se sabe que dentro de estas dos visiones, es muy probable encontrar posiciones con distintos matices. La intención, entonces, es poder describir las concepciones de los docentes respecto de este aspecto de la evaluación en función de los matices encontrados al analizar sus respuestas a esta pregunta. 


\subsection{Procedimiento}

Recolección de datos

Se realizaron entrevistas que se registraron en formato digital y duraron aproximadamente una hora (cuestionario completo de 23 preguntas). Las mismas se llevaron a cabo en un marco natural, abierto y en tono de conversación para permitir que la posición de los entrevistados emergiera de manera espontánea. Se estudió el conjunto de las respuestas dadas por todos los sujetos que compusieron la muestra y se extrajeron categorías de respuestas sobre sus creencias acerca de “¿qué se evalúa?”, ¿cómo se evalúa? y ¿qué función tiene la evaluación en el aprendizaje?”.

Análisis de datos

En primer lugar se extrajo un número limitado de conceptos que permitieron delinear las dimensiones de análisis. Para la identificación de categorías se utilizó el método comparativo constante. (STRAUSS; CORBIN, 1997) Se comenzó por realizar un examen independiente de los datos por parte de los investigadores responsables del trabajo y un proceso iterativo de identificación y definición de categorías de respuestas. Para obtener una visión global de las concepciones de los docentes frente a la enseñanza, las entrevistas se escucharon varias veces, se compararon entre sí y se interpretaron en un sentido amplio y no en función de los matices locales del discurso de cada sujeto. La categorización comenzó con la formación tentativa de orientaciones en la que los casos que parecían similares se consideraron en una misma categoría siempre que fueran suficientemente diferentes de otras categorías nacientes. Una vez finalizado el examen independiente, se realizó un proceso de re-categorización conjunto, extrayendo respuestas a medida que surgieron agrupaciones alternativas, continuando el proceso hasta que las mismas se estabilizaron. Se concluyó la etapa describiendo las respuestas encontradas tanto para los aspectos “¿qué se evalúa?”, ¿cómo se evalúa?, como para el relacionado con “¿qué función tiene la evaluación en el aprendizaje?".

\section{I RESULTADOS Y DISCUSIÓN}

Descripción de las categorías encontradas

Se describen las categorías de respuesta (concepciones) obtenidas para cada dimensión, una vez finalizado el análisis de las entrevistas.

\section{Dimensión 1: Concepciones respecto de qué se evalúa}

En el tipo de respuesta R1, se ubicaron aquellas concepciones de docentes que, mediante la evaluación, buscan constatar la incorporación de información por parte del estudiante, en términos de recuerdo de hechos y procesos algorítmicos cerrados y aislados. Aparecen expresiones como, por ejemplo:

"Me doy cuenta que un estudiante aprendió porque incorporó los conceptos que intento transmitir..." 
"[Me doy cuenta que un estudiante aprendió] cuando puede resolver otra situación que difiere de la anterior donde no sólo se cambiaron los números. Cuando puede aplicar los conocimientos a un problema global en el cual no está siguiendo un tema puntual de la guía sino un problema que abarca distintos tópicos".

El tipo de respuesta R2 incluye a la anterior, pero los docentes aquí involucrados buscan un adicional relacionado con la formación profesional. Aspectos vinculados con el desarrollo de ciertas competencias profesionales aparecen en este discurso:

"Yo para evaluar tomo dos parciales, los trabajos prácticos y un examen oral, final, un totalizador de toda la materia, donde charlamos un rato de todo lo que vimos en la materia. Esto lo hago no solo pensando en la asignatura sino en aspectos de un ingeniero, de lo que va a tener que hacer un ingeniero..."

"La forma más directa [de saber que un alumno aprendió] es replicar en una evaluación un problema al que creemos que se va a enfrentar en su vida profesional"

El tipo de respuesta R3 hace referencia al discurso de algunos docentes que no aportó información a la dimensión evaluada: respuestas evasivas o referencias inconexas.

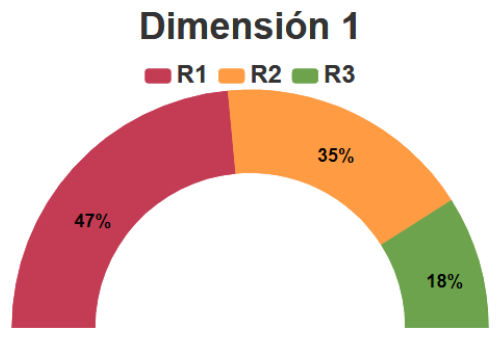

Figura 1. Distribución de respuestas para la dimensión “¿qué se evalúa?”.

\section{Dimensión 2: Concepciones respecto de cómo se evalúa}

En el tipo de respuesta $\mathrm{R} 1$, se ubicaron aquellas concepciones de docentes que tiene en cuenta a la evaluación como para calificar y acreditar los resultados. Hacen hincapié en el conocimiento memorístico y enfatizan en el producto del aprendizaje (lo observable). El desempeño de los estudiantes, sus recorridos, sus superaciones, no son tenidos en cuenta por estos docentes. El principal instrumento es el examen de lápiz y papel, y las instancias se reducen a un número acotado.

"Los evaluamos con dos parciales. Los parciales son escritos. La forma de chequear [que aprendieron] es el parcial, para ser justos con todos".

"El escrito es lo que está escrito y ahí hay que remitirse, a las pruebas escritas nada más".

"La única manera que tenemos nosotros en la universidad es a través de los 
exámenes, no hay otra."

Los docentes cuyas respuestas se agrupan en el tipo R2, siguen poniendo énfasis en la evaluación como un resultado, pero tienen en cuenta ciertos elementos del proceso. Estos docentes incorporan algunas herramientas para este seguimiento: uso de rúbricas para facilitar el monitoreo de los aprendizajes de los estudiantes, utilización de diferentes estrategias de evaluación, uso de la evaluación para la regulación del proceso de enseñanza.

"Yo no pongo puntaje [en los parciales], pongo "aprobado" o "desaprobado", y me guardo algún registro de esa performance. Es laborioso, pero pongo en un casillero un "muy bien" o alguna nota de eso...Entonces, [al final del curso] tengo cómo rinden, cómo escriben y cómo se expresan".

El tipo de respuesta R3, como para la dimensión anterior, hace referencia al discurso de algunos docentes que no aportó información a la dimensión evaluada.

\section{Dimensión 2}

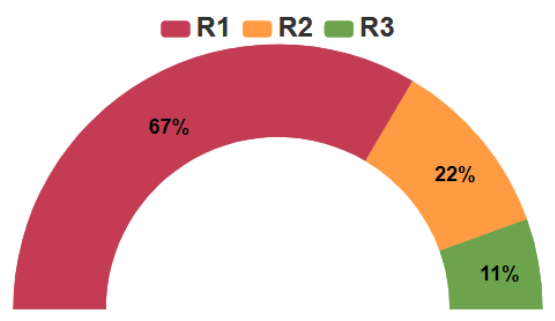

Figura 2. Distribución de respuestas para la dimensión “¿cómo se evalúa?”.

\section{Dimensión 3: Concepciones respecto de qué función tiene la evaluación en el}

\section{aprendizaje}

Las concepciones que se ponen de manifiesto aquí podemos resumirlas en la mirada formadora o en la mirada acreditadora de la evaluación. (SANMARTí, 2011) Los docentes con respuestas del tipo R1 consideran que la evaluación solo mide resultados y se la desvincula completamente de los procesos de enseñanza y de aprendizaje. Estos docentes corrigen y devuelven las producciones corregidas, sin tener en cuenta que el que debe corregir los errores es quien los comete, es decir, el estudiante.

"Siempre les digo que vean el examen...somos humanos y podemos cometer errores, no solo en la corrección sino en la suma de los puntajes. En un sistema promocional la nota es importantísima"

"Al final uno termina calificando por la evaluación".

"Si hubo algún problema que fue general, generalmente hacemos algún comentario que normalmente lo hago en la clase teórica que es donde van más alumnos". 
Aquellos docentes que ven otra función en la evaluación más allá de la acreditación tienen respuestas del tipo R2. Estos docentes le asignan una función formadora y la consideran como otra instancia del aprendizaje. Algunos proponen diferentes estrategias para no caer en evaluaciones tradicionales que solo miden un resultado, pero terminan haciendo un "promedio" de todas las calificaciones individuales.

"A veces el alumno llega a ver el examen y explica que después de entregarlo llegó a su casa y se dio cuenta que se había equivocado, entonces indica de qué manera lo resolvería. Eso ayudó al conocimiento del alumno. Eso manifiesta una intención del alumno de que sepamos que él estudió, y eso me parece valioso".

Nuevamente, las respuestas agrupadas como R3 incluyen los casos en los que el discurso del docente no aporta información para la dimensión considerada.

\section{Dimensión 3}

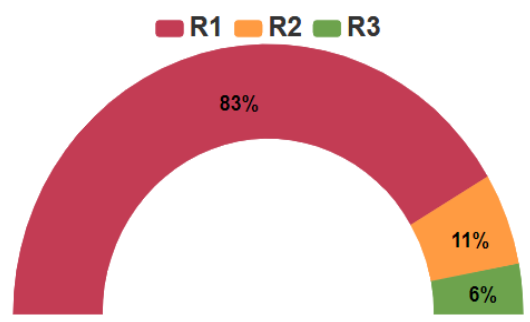

Figura 3. Distribución de respuestas para la dimensión “¿qué función tiene la evaluación?”

\section{I CONCLUSIONES}

El objetivo de esta investigación fue desarrollar un análisis preliminar, pero sistemático, de las concepciones sobre la evaluación que poseen los profesores entrevistados de diferentes asignaturas de ingeniería. Dicho análisis, llevado a cabo a partir de la indagación de las opiniones que tienen sobre sus prácticas, arrojó como resultado que conciben a la evaluación como una estrategia para medir la habilidad alcanzada por los estudiantes para la reproducción del conocimiento y de procedimientos cerrados, que implican sólo la aplicación de algún tipo de algoritmo, más que como un instrumento que favorezca el desarrollo de la capacidad para reestructurar o transformar el conocimiento que le han 'dado'.

Se esperaba encontrar una categoría que describiese una concepción de evaluación más cercana a las teorías actuales desarrolladas en el campo de la enseñanza de las ciencias, cuyo objetivo sea buscar que el estudiante tenga la oportunidad de demostrar la habilidad de integrar, transformar y usar el conocimiento internalizado en función de resolver problemas del campo profesional, por ejemplo; sin embargo, no se hallaron este tipo de 
opiniones. Si bien aparecen algunos casos de docentes que conciben una evaluación que favorece la construcción y transformación del conocimiento, esta idea es fundamentalmente de carácter voluntarioso, no apareciendo menciones de prácticas concretas ligadas a la evaluación que favorezcan este tipo de aprendizaje. Sólo se mencionan unos pocos hechos aislados y lo hacen los docentes que están a cargo de las asignaturas de final de carrera, vinculadas a la práctica profesional.

Estos primeros resultados son relevantes, ya que se observa que de los tres niveles de concepciones descriptas por otros autores (tradicional, intermedia e innovadora) sobre evaluación, el cuerpo docente entrevistado manifiesta en gran medida una concepción tradicional y en menor grado una concepción intermedia. No obstante, cabe destacar que se necesitan más investigaciones para documentar y entender las relaciones entre las creencias sobre enseñanza, aprendizaje y evaluación en el campo de la enseñanza de la ingeniería.

También resulta importante resaltar que, más allá del análisis realizado se pudo observar que las entrevistas promovieron un proceso de reflexión y análisis de las propias prácticas docentes que podría contribuir a mejorar el proceso de formación de conocimiento en los estudiantes.

Por último, la descripción realizada de las concepciones proporciona un estado de situación a partir del cual es posible proyectar el diseño de diferentes propuestas de formación pedagógica para aquellos docentes de ingeniería que estén interesados en el tema. Resignificar las concepciones de los docentes sobre la enseñanza y el aprendizaje de la ingeniería hacia formatos más actuales, es un objetivo muy a largo plazo dada la resistencia que hay a dedicar tiempo a este tipo de cuestiones y, particularmente, a plantearse nuevas preguntas respecto de algo tan naturalizado como lo es la evaluación. Es probable que cumplir con este objetivo requiera un cambio de ideas que, para muchos docentes, resulte equivalente a experimentar un cambio conceptual (STRIKE; POSNER, 1992), que es un cambio fundamental en sus supuestos sobre la enseñanza y en sus valores. (SAMUELOWICZ; BAIN, 2001).

\section{REFERENCIAS}

DEL HOYO, J.; HORMAIZTEGUI, M. E. V.; MASSA, P.; FANOVICH, M. A.; MORO, L. E.; BUFFA, F.; GARCÍA, M. B. Categorización de las concepciones de los docentes universitarios de Ingeniería sobe "qué se enseña" y "para qué se enseña", Actas del III Congreso Argentino de Ingeniería CADI2016, (pp. 478-488).Resistencia, Argentina. 2016.

GAIRIN, J.; SANMARTÍ, N. La evaluación institucional, Ministerio de Educación de la Nación, Argentina. 1998.

KEMBER, D. Teaching beliefs and their impact on students' approaches to learning, Dart, $B$. and Boulton-Lewis, G. (coord.), Teaching and Learning in Higher Education. Camberwell, Vic: ACER Press. 1997. 
LIMÓN, M. The domain generality specificity of epistemological belief. A theoretical problem, a methodological problem or both?, International Journal of Educational Research 45, 7-27. 2006.

PECHARROMÁN, I.; POZO, J. I. ¿Cómo sé que es verdad?: Epistemologías Intuitivas de los Estudiantes sobre el Conocimiento Científico, Investigações em Ensino de Ciências, 11 (2),153-187. 2006.

PONTES PEDRAJAS, A.; POYATO LÓPEZ, F. J.; OLIVA MARTíNEZ, J. M. Concepciones Sobre Evaluación en la Formación Inicial del Profesorado de Ciencias, Tecnología y Matemáticas, Revista Iberoamericana de Evaluación Educativa, 9 (1), 91-107. 2016.

PROSSER, M., MARTIN, E., TRIGWEL, K., RAMSDEN, P.; LUECKENHAUSEN, G. Academics experiences of understanding of their subject matter and the relationship to their experiences of teaching and learning, Instructional Science, 33, 137-157. 2005.

SAMUELOWICZ, K.; BAIN, J.D. Revisiting academics' beliefs about teaching and learning, Higher Education 41, 299-325. 2001.

SAMUELOWICZ, K.; BAIN, J.D. Identifying academics' orientations to assessment practice, Higher Education 43, 173-201. 2002.

SANMARTÍ, N. Evaluar para aprender, evaluar para calificar, Caamaño, A. (coord.), Didáctica de la Física y la Química, (p. 193-211). Ministerio de Educación Cultura y Deporte, Secretaría General Técnica-Graó: Barcelona. 2011.

SCOULLER, K. How assessment-driven are students really?, Rust, C. (coord.), Improving Student Learning: Improving Student Learning Outcomes. Oxford: The Oxford Centre for Staff and Learning Development. 1999.

STRAUSS, A.; CORBIN, J. Grounded Theory in Practice. London: Sage. 1997.

STRIKE, K. A., POSNER, G. J. A revisionist theory of conceptual change, Duschl, R.A. y Hamilton, R.J. (coord.), Philosophy of science, cognitive psychology, and educational theory and practice, (p. 147176). State University of New York Press: Nueva York. 1992.

THOMAS, P.R.; BAIN, J.D. Contextual dependence of learning approaches: the effects of assessments, Human Learning 3, 227-240. 1984.

VILANOVA, S. L.; MATEOS-SANZ, M. del M.; GARCÍA, M. B. Las concepciones sobre la enseñanza y el aprendizaje en docentes universitarios de ciencias, Revista Iberoamericana de Educación Superior, 3 (2), 53-75. 2011. 


\section{ÍNDICE REMISSIVO}

\section{A}

Atenção primária 58

Avaliação 23, 33, 35, 36, 45, 69, 73, 83, 96, 103, 141, 158, 179

B

Base Nacional Comum Curricular 40, 41, 43, 48, 51

C

\section{Células-Máter 181}

Competências socioemocionais 40, 45, 46, 47, 48, 50, 51

Concepções 49, 65, 125, 158, 202, 210

Criança $22,25,26,46,52,53,54,55,56,71,72,73,118,119,120,121,122,123,129$, $137,169,170,171,172,173,174,175,176,177,178,179,180,215$

Crianças Refugiadas 52, 53, 54, 55, 56

Criminalidade 20, 21, 24, 28

\section{D}

Desenvolvimento Cognitivo 70, 118, 120, 121, 122, 172, 212

Desenvolvimento Humano 1, 2, 3, 4, 10, 21, 26, 33, 100, 180, 210

Desenvolvimento Sustentável 3, 77, 78, 80, 90, 95, 96, 97

Dificuldade de aprendizagem 35

Digitalização 146, 148, 149, 150, 151, 152, 154, 155

Diversidade $3,13,16,17,18,23,46,48,62,124,129,130,131,194,198,211,212$

E

Educação 2, 9, 1, 2, 3, 4, 5, 6, 7, 8, 9, 10, 11, 12, 14, 15, 16, 17, 18, 19, 22, 23, 24, 26, 27, $28,30,33,34,40,41,42,43,44,45,46,47,48,49,50,51,52,55,56,57,58,59,60,62$, $63,64,65,66,67,68,69,70,71,72,73,74,75,76,77,78,79,80,81,83,84,87,89,90$, $91,95,96,98,99,100,101,102,104,106,107,108,109,112,113,114,116,117,118$, $119,123,124,125,129,138,139,140,141,142,143,144,145,146,147,148,150,151$, $152,153,154,155,169,170,171,172,173,175,179,180,186,187,188,189,190,194$, 195, 196, 197, 198, 199, 200, 201, 202, 203, 204, 205, 206, 207, 208, 209, 210, 212, 213, $214,215,216,217$

Educação Básica 41, 44, 47, 125, 139, 189, 216

Educação de Refugiados 52

Educação Infantil 69, 70, 71, 72, 73, 74, 75, 118, 119, 125, 169, 170, 175, 179, 180, 208 


\section{Empresas Educativas 146}

Engenharia 76, 78, 79, 80, 81, 82, 96, 158, 216, 217

Ensino 9, 7, 8, 13, 15, 16, 17, 26, 33, 35, 36, 38, 39, 40, 43, 45, 48, 49, 50, 51, 71, 76, 77, $78,80,81,95,96,97,98,99,100,102,103,104,105,106,107,108,109,110,112,113$, $114,115,116,121,122,123,124,125,129,131,133,134,135,136,137,140,142,143$, $145,148,150,151,153,155,158,168,169,170,171,172,173,174,175,178,179,187$, 188, 189, 190, 191, 192, 195, 198, 199, 200, 202, 205, 207, 208, 209, 210, 211, 214, 216, 217

Ensino universitário 77

Esporte 20, 21, 24, 25, 26, 27, 28, 29, 30, 31, 32, 33, 34, 216

Experimento didático-pedagógico 98, 99, 101, 103

Extensão 76, 77, 78, 79, 80, 81, 82, 84, 87, 95, 96, 97, 114, 117, 151, 152, 189

\section{$\mathbf{F}$}

Fitoesteídrico 181, 182, 183

Formação $1,2,4,5,6,8,9,11,12,17,18,21,30,35,40,41,43,44,45,49,50,58,65$, $68,69,70,71,72,73,79,80,81,96,97,114,117,140,142,145,146,154,155,169,173$, $174,175,178,179,180,186,187,188,189,190,191,194,195,196,198,199,203,209$, $210,211,213,214,215,216,217$

Formação de pedagogos 186

Formação Docente 169, 179

G

Gamificação 98, 99, 100, 101, 103, 104

Gerenciamento de resíduos sólidos 76, 77, 80, 81, 82, 83, 87, 90, 96

H

Hipofisário 181, 182

Histórias em Quadrinhos 124, 125, 127, 137

I

Inclusão em educação 123

Indígenas 124, 127, 128, 129, 130, 131, 134, 136, 137

Informação $19,29,47,53,54,80,85,106,115,139,140,141,143,145,146,147,148$, 149, 150, 151, 173, 174, 175, 176, 197, 198, 199, 202, 205, 207, 208, 209, 210, 211, 212, 213

Inovação $144,146,213$ 
Juventude 20, 22, 24, 34, 43, 117

L

Leitura do mundo $1,2,4,5,6,7,8,9,12$

Linguagem $6,26,42,69,70,71,72,73,74,75,110,114,119,121,122,124,125,126$, $137,172,173,195$

\section{M}

Meio Rural 58, 60, 63, 67, 68

Metodologias lúdicas 106

Metodologias Participativas 106, 108, 113, 116

Método Montessori 169, 170

N

Neurociência Educacional 169, 170, 172

$\mathbf{P}$

Pesquisa-intervenção 106, 108, 114, 115, 117

Professores 13, 16, 17, 18, 27, 30, 37, 42, 44, 49, 69, 71, 73, 74, 78, 81, 83, 95, 109, 112, $116,122,129,139,140,141,142,143,144,145,153,155,158,175,180,188,189,190$, 194, 195, 196, 200, 201, 204, 208, 209, 210, 211, 213, 214, 216, 217

Projeto Social 20, 27, 28, 29, 30, 31, 32

Psicopedagogia $74,118,121,176,216$

$\mathrm{R}$

Reforma Empresarial da Educação 40, 42, 43, 51

Rondônia 124, 127, 128, 129, 131, 132, 133, 134, 135, 136, 137

$\mathrm{s}$

Saúde da população rural 58

Síndrome de Down 118, 119, 123

$\mathbf{T}$

Tecnologias 43, 47, 109, 139, 140, 141, 142, 143, 144, 145, 146, 147, 148, 150, 154, 155, 199, 202, 203, 205, 208, 209, 210, 212, 213, 214, 217

TIC 146, 147, 148, 150, 151, 152, 155, 208

Trabalho de Conclusão de Curso 13, 186, 187, 190, 191, 192, 193, 194

Transgressão $13,14,17$ 
Tritalâmica 181

$\mathbf{U}$

Universidade $1,9,12,13,33,34,40,52,58,76,77,78,79,81,83,88,91,95,96,97,106$, $117,118,123,124,129,131,133,134,135,136,139,153,158,169,186,187,208,214$, 216 


\section{Educação e a}

Apropriação e

Reconstrução do

\section{Conhecimento}

\section{Científico}




\section{Educação e a}

Apropriação e

Reconstrução do

\section{Conhecimento}

\section{Científico}

